\title{
Functional foods and health: a US perspective
}

\author{
J. A. Milner* \\ Nutritional Science Research Group, Division of Cancer Prevention, National Cancer Institute, \\ 6130 Executive Boulevard, Suite 3164, Rockville, MD 20892, USA
}

\begin{abstract}
Linkages between diet habits and the quality of life continue to surface on numerous fronts. Collectively these epidemiological, pre-clinical and clinical studies provide rather compelling evidence that numerous essential and non-essential dietary components are capable of influencing growth, development and performance and disease prevention. Scientific discoveries and widespread interest in the potential medicinal benefits of foods and food components have fostered a variety of content, health and structure-function claims. Unfortunately, defining the ideal diet is complicated by the numerous and diverse components that may influence biological processes. Inconsistencies in the literature may reflect the multi-factorial nature of these processes and the specificity that individual dietary constituents have in modifying genetic and epigenetic events. New and emerging genomic and proteonomic approaches and technologies offer exciting opportunities for identifying molecular targets for dietary components and thus determining mechanisms by which they influence the quality of life. All cells have unique 'signatures' that are characterized by active and inactive genes and cellular products. It is plausible that bridging knowledge about unique cellular characteristics with molecular targets for nutrients can be used to develop strategies to optimize nutrition and minimize disease risk.
\end{abstract}

Diet: Cancer: Polymorphism: Genes: Phytochemicals

\section{Introduction}

There is little doubt that nutrition and health are intimately linked. For generations, people have believed that foods could do more than merely provide energy. Beliefs in the medicinal properties of foods were highlighted in a number of the early writings of mankind. Hippocrates is frequently quoted as saying: 'Let food be thy medicine and medicine be thy food'. Epidemiological, pre-clinical and clinical studies continue to provide fundamental insights into the dynamic relationships between nutrients - defined here as any substance in the diet that brings about a physiological effect — and health. Today, claims about the ability of foods and food components to reduce disease risks or enhance the quality of life continue to captivate our lives. In North America, passionate discussions about foods and beverages are commonplace between friends, relatives and even complete strangers.

\section{Unprecedented opportunities}

Inappropriate nutrition is a primary factor in unattained genetic potential, reduced physical and cognitive performance, and increased risk of some diseases. Unquestionably, strategies that optimize nutrition by the use of foods or supplements are highly commendable and considered by many to be appropriate for improving the overall quality of life. The importance of such strategies is emphasized by the recognition that about one-third of all cancers are related to dietary habits. Actually, more than half of the deaths occurring in the United States correlate with food patterns (National Center for Health Statistics, 1997). Many in North America believe that modifying their diet and the use of supplements are two of the most important options available to reduce health care costs and improve personal health (Eisenberg et al. 1998; Gilbert, 1999; Aldana, 2001).

In spite of a growing number of studies, workshops and conferences devoted to understanding the dynamic relationship existing between nutrition and disease, it remains virtually impossible to determine who will and will not benefit most, if at all, from dietary intervention. What is recognized is that the effect of food on health varies considerably among people, both in terms of its direction and magnitude (Clydesdale, 1998; Milner, 2000). Part of the difficulty in determining those who might benefit arises from the incredibly complex chemical

\footnotetext{
Abbreviations: ATBC, Alpha-Tocopherol, Beta-Carotene Cancer Prevention Study; CGAP, Cancer Genome Anatomy Project; FDA, Food and Drug Administration; ILSI, International Life Sciences Institute.

* Corresponding author: Dr J. A. Milner, fax +1 301480 3925, email milnerj@mail.nih.gov
} 
composition of the food supply (Leclercq et al. 2001). Likewise, increasing evidence points to genetic variability as a significant determinant of the response to foods and food components. While delineating the most effective diet for an individual is a daunting task, recent scientific discoveries as reviewed briefly below reinforce the belief that a personalized dietary intervention approach to health promotion and disease prevention is feasible.

\section{Functional foods: a health perspective}

The intriguing term 'functional foods' has arisen from a general belief in the health benefits of foods. While this term has no legal meaning, it nevertheless signifies a proactive appreciation that some foods may confer health benefits (Milner, 2000). While functional foods have been defined in various ways, the International Life Sciences Institute (ILSI) North America defines them as: 'Foods, that by virtue of physiologically active food components, provide health benefits beyond basic nutrition'. Classifying which foods fall within the health benefits category and which do not has been an interesting challenge for some, especially since many factors can influence the overall response to a food including the quantity consumed, accompanying foods ingested, duration of exposure, physiological state, etc. At this point, it is best to indicate that all foods are probably functional in 'some' capacity and that, under specific circumstances, some may provide immediate and/or long-term benefits.

Widespread interest in functional foods continues to abound in North America among scientists, legislators and consumers. About $60 \%$ of adults residing in the United States are believed to select foods for health purposes, regardless of their age or gender (http://www.IFIC. org). While younger individuals may be selecting foods for mental and physical performance, older individuals appear to be selecting foods for their potential merits in reducing disease risk or improving the quality of life. Regardless of why foods are chosen, it is refreshing to have a positive proactive belief, since during the past few decades campaigns to alert people about diet and health issues have largely been negative.

\section{Market value of functional foods in the United States}

Increasing consumer demand for healthful foods and beverages, coupled with scientific discoveries about the physiological consequences of selected foods and ingredients, has fostered the development of an active functional foods and beverage market. Some of these products certainly extend beyond conventional fortified items because they contain specific ingredients designed to have health or structure-function benefits for the consumer.

Statistics about the size of the US natural foods market vary considerably depending on the source of information and which products are included or excluded, as evident by a recent report entitled 'The Natural/Organic Food Market in the United States' (http://atnriae.agr.ca/info/us/ e3164.htm\#MARKET OVERVIEW). Many estimate that natural and organic foods account for annual sales of between $\$ 16.3$ billion and $\$ 29.7$ billion, which undeniably represents a large variance. The market value of functional foods is also largely ill defined. Frost \& Sullivan (http:// www.food.frost.com) suggest the total market for functional ingredients, functional foods, functional beverages, dietary supplements and foods for special dietary use is approximately $\$ 50$ billion. Trade publications for the food industry in 2000 indicated that sales of natural products including foods, beverages and supplements grew by about $7 \%$ over the previous year.

\section{Health and structure-function claims in North America}

The United States' Federal Food, Drug and Cosmetic Act does not provide a statutory definition of functional foods. Thus, the Food and Drug Administration (FDA) has no authority to establish a formal regulatory category for such foods. The primary determinant of the regulatory status of these foods is thus their intended use. The distinction between a food and a drug has been blurred because of recent scientific findings suggesting that the former may have some medicinal properties. Nevertheless, according to the FDA, a drug is a substance that is used to prevent, treat, cure or mitigate disease. Foods, on the other hand, and according to Webster's Dictionary, are defined as protein, carbohydrates, fats, minerals, vitamins and other supplementary substances that nourish and sustain life. According to the FDA, a food is defined as: 'Articles used for food or drink for man or other animals, chewing gum, and articles used for components of any such article'.

In the United States, food claims can take a number of approaches, i.e. those related to content, health or to structure-function. In marketing these foods, manufacturers may come under one of several existing regulatory options. The first decision manufacturers will make that will help determine their product's regulatory status is whether the product is a food or a drug. Thus, manufacturers and retailers have a range of legal and regulatory categories into which their products may be classified. Fifteen health claims have been approved for manufacturers to describe the relationship between a food substance and a disease or health-related condition. Some of the broad areas

Table 1. Areas associated with health claims in the United States

Calcium and osteoporosis

Dietary lipids and cancer

Sodium and hypertension

Dietary saturated fat and cholesterol and the risk of CHD

Fibre-containing grain products, fruits and vegetables and cancer

Fruit, vegetables and grain products that contain fibre, particularly soluble fibre, and the risk of CHD

Fruits and vegetables and cancer

Folate and neural tube defects (1996)

Dietary sugar alcohols and dental caries (1996)

Soluble fibre from certain foods and the risk of CHD (1997)

Soya protein and the risk of CHD (1999)

Stanols and heart disease

$\mathrm{CHD}$, coronary heart disease. 
addressed by these health claims are presented in Table 1 . Health claims may arise in three ways. First, the 1990 Nutrition Labeling and Education Act allows the FDA to authorize a health claim for a food or dietary supplement based on the FDA's careful review of the scientific literature. Second, the 1997 Food and Drug Administration Modernization Act allows a health claim to be made for a food based on an authoritative statement of a scientific body of the US government or the National Academy of Sciences. And third, the 1999 court decision in the case of Pearson vs. Shalala allows certain qualified health claims to be used. The relatively small number of health claims for foods that have been approved serves to emphasize the incomplete or inconsistent information that precludes firm conclusions.

Structure-function claims have been allowed for foods under the US Federal Food, Drug and Cosmetic Act, but because they are allowed differently for dietary supplements and conventional foods their use is stirring considerable controversy in the USA. Some current uses and controversies about structure-function claims focus on how best to identify an action of the food or bioactive food component without simultaneously imply a health or drug outcome. Thus, structure-function statements are often vague and imply the food or components helps maintain normalcy, whatever that might signify.

Historically, the Food Regulations under the Canadian Food and Drugs Act were developed with the objectives to ensure that food that was sold was safe and nutritious and for the prevention of fraud. The recognition of the health effects of various food constituents has sparked legislative interest in functional foods. In Canada foods containing the beneficial ingredients, whether naturally occurring or as a result of addition of an isolated component, are termed 'functional foods'. The proposed Health Canada definition of a functional food (http:// www.hc-sc.gc.ca/main/drugs/zfiles/english/ffn/ffdscdoc_e. $\mathrm{html}$ ) is one that is: "similar in appearance to, or may be, a conventional food, is consumed as part of a usual diet, and is demonstrated to have physiological benefits and/or reduce the risk of chronic disease beyond basic nutritional functions'. Currently a proposal is pending that would allow health claims in Canada.

Regardless of the claims that are allowed, consumers are eager to learn more truthful information about food, food components and health. To assist in clarifying the issue, ILSI North America's Technical Committee on Food Components for Health Promotion developed in 1997 a Road Map as a strategy to 'Improve the Health of the Public through Consumer Acceptance of Safe Food Products that Provide Significant Health Benefits'. A number of approaches were identified to accomplish this goal including: the creation of a comprehensive science base, the promotion of public trust, the development of consumer preferred functional foods, the optimization of a regulatory framework and the creation of marketplace incentives to develop functional foods. Because of the scientific and legal mandates involving organizations such as ILSI, it was apparent that other groups were needed if progress was truly going to be made. Delightfully, progress has occurred during the past four years in not only showcasing this area but also with building a number of important and effective linkages. Undeniably the database regarding foods and their components as factors in health has continued to blossom during this period. Likewise, new and exciting linkages have surfaced with various groups with a commitment to research and outreach about foods and health. The turn of the twentyfirst century was a point to not only reflect on what had been accomplished by the Road Map, but to rekindle interest in foods and bioactive components as a continuing focus. While the exact plan remains to be resolved, key components will probably be to stimulate fundamental science to evaluate the health effects of functional foods, develop a framework to utilize science for decision making and optimize the scientific impact of these undertakings through key collaborations.

\section{Numerous bioactive food components may be involved}

Numerous foods are already associated with health promotion and disease prevention. The diversity of these foods suggests a variety of components may be involved. It has been estimated that about 25000 different chemical compounds occur in fruits, vegetables and other plants eaten by man. To date, more than 500 compounds have been identified as potential modifiers of the cancer process. Both essential and non-essential allelochemicals occurring in plants, as well as zoochemicals found in animal products, may be physiologically important modifiers of a host of biological processes. Compounds encompassing such diverse categories as carotenoids, dithiolthiones, flavonoids, glucosinolates, isothiocyanates, allyl sulfhydryls and fermentable fibres have been found to influence a variety of cellular processes that would be expected to influence health (Hasler et al. 2000; Milner, 2000; Prosky, 2000; Go et al. 2001). Many have been reported to modify the redox status of cells and therefore may have far-reaching implications as determinants of health and well-being (Diplock et al. 1998; Clarkson \& Thompson, 2000; Roberfroid, 2000). Numerous reviews extolling the merits and/or possible risks of numerous bioactive food components have been presented in recent years (Potter, 1997; Abdulla \& Gruber, 2000; Gill \& Cross, 2000; Milner, 2000).

\section{Moving beyond observational studies}

While observations about dietary bioactive food components and health are exceedingly tantalizing, the future of nutrition research probably resides in the ability to move beyond these studies to a more probing and molecular approach that will allow for tailored recommendations to individuals (Greenwald \& Milner, 2001). DellaPenna (1999) coined the term 'nutritional genomics' to describe work at the interface of plant biochemistry, genomics and human nutrition aimed at understanding and manipulating nutrient reactions and interactions at the molecular or genomic level. For the purposes of this review, nutritional genomics refers to the study of any genetic or epigenetic interaction with a nutrient that leads to phenotypic changes. Unquestionably, the study of nutritional genomics offers 
the potential to identify definitively which components in foods influence growth, development and health, and to clarify their specific mechanisms of action. About 26000 to 38000 genes were proposed in the first draft of the human genome. While this is only about double the number found in the fruit fly and worm, it points to the importance of the expression of these genes and to their regulation (Paabo, 2001). A web site (http://www. cgap.nci.nih.gov/), developed jointly by the National Cancer Institute and the National Library of Medicine, was part of the recently launched Cancer Genome Anatomy Project (CGAP). The database offers scientists a powerful new tool to study how various factors including dietary components might alter a host of cellular events. Queries to the CGAP database are examined quickly through a gene index system that a few years ago might have taken years or even lifetimes to compile. Genomic data for man and mouse, including expressed sequence tags, gene expression patterns, single nuclear polymorphisms, cluster assemblies and cytogenetic information, are included. In addition to genetic information, this web site contains informatics tools to query and analyse the data and information on methods and resources for reagents developed by the project. All CGAP resources - including cDNA libraries, clones and sequence data - are publicly accessible to scientists.

Rather compelling evidence already exists revealing that a variety of nutrients can influence genetic and epigenetic processes that determine cellular metabolism, differentiation and apoptosis (Bradlow et al. 1999; Knowles \& Milner, 2000; Pan et al. 2000; Blanchard et al. 2001; Merrill et al. 2001). It is known that cell homeostasis is regulated by a finely controlled balance among proliferation, growth arrest, differentiation and apoptosis (programmed cell death). Disruption in this balance can lead to profound phenotypic changes ranging from growth suppression to the transformation of normal into neoplastic cells. Dysregulation of apoptosis is frequently accompanied with the pathogenesis arising from a wide array of conditions including neurodegeneration, autoimmunity, heart disease, cancer and others. Several nutrients are already identified as factors influencing cellular homeostasis. For example, while vitamin A has repeatedly been shown to be involved with differentiation, other nutrients such as vitamin D and lignan may also play a role (Ward et al. 2000; Gray et al. 2001; Lee \& Pelletier, 2001). Since various nutrients can influence the same process, it becomes of increasing importance to understand the ideal balance of these nutrients that brings about a desired effect and whether a shift in this balance leads to changes in physiological processes and/or phenotypic characteristics. These dynamic interactions are not limited to differentiation but are also evidenced by the ability of diverse nutrients such as plant sterols, Se and butyrate arising from fermentable fibres to promote apoptosis in intestinal cells (Awad \& Fink, 2000; Chapkin et al. 2000; Schrauzer, 2000). One of the major issues to be clarified in the future is the minimum quantity of these dietary components required to bring about phenotypic effects and if genetic differences within tissues determines their physiological consequences. Unfortunately, far too often many pre-clinical studies utilize concentrations of bioactive food components that would be virtually impossible to achieve with typical daily eating behaviours. Historically, evidence has surfaced that cells are effective in acclimatizing to insults resulting from exposures to excessive quantities of nutrients (Fafournoux et al. 2000; Jackson, 2000). It remains to be determined if intermittent intake of nutrients offers advantages or if sustained intake is a method to promote health and well-being.

Excesses are not the only way that nutrients may change cellular events. Caloric restriction is associated with a reduction in a number of age-specific chronic diseases (Turturro et al. 1994). Evidence already exists that the transcriptional silencing of selected genes by DNA methylation plays a crucial role in ageing and a number of diseased states (Issa, 2000). Evidence with yeast indicates that caloric restriction can lead to the silencing of a variety of genes (Guarente \& Kenyon, 2000).

While the study of nutritional genomics is still in its infancy, it is starting to reveal that nutrient excesses and deficiencies can bring about a host of genomic and proteonomic changes. Regardless of whether the molecular target is at the transcription, translation or post-translational level, the net result is an up- or down-regulation of specific gene products. Unravelling the multitude of interactions among nutrients with these key events makes the challenge of nutritional genomics extremely daunting. Inter-individual differences probably reflecting genetic polymorphisms may mask the response to an individual nutrient and thereby complicate this undertaking to an even greater degree (Hegelea et al. 1997; Hegele, 1998; Rapuri et al. 2001). In some cases nutrients that are generally thought to be protective may actually increase risk (HilakiviClarke et al. 1999; Ross, 2000). Deciphering the dynamic relationship between dietary components and genes is fundamental to optimizing health. With the information gained, it should be possible to determine why inconsistencies occur in the nutrition and health literature and to develop meaningful and tailored strategies to assist individuals.

Complementary and overlapping mechanisms appear to account for the response to bioactive food components. These biological responses encompass such diverse functions as serving as an antioxidant, promoting the activity of detoxification enzymes, blocking carcinogen formation and metabolism, shifting hormonal homeostasis, retarding cell division, and inducting apoptosis. Since more than one of these processes may be influenced simultaneously, it is difficult to determine which is most important in explaining any phenotypic changes.

Some of the most compelling evidence that diet can influence the cancer process comes from the intervention study by Clark et al. (1996) with Se yeast as a supplement. Based on these findings and a host of pre-clinical studies, the National Cancer Institute and a network of researchers known as the Southwest Oncology Group initiated the largest-ever prostate cancer prevention study, The Selenium and Vitamin E Cancer Prevention Trial, to determine if these two dietary supplements can offer protection (http://newscenter.cancer.gov/pressreleases/ SELECTQandA.html). Although much of the attention 
on Se during the past decade has focused on its antioxidant activity, this trace element is known to bring about a diverse set of biological effects including suppressing cell proliferation, enhancing immunocompetence, blocking carcinogen metabolism, and induction of apoptosis. By identifying which one of the events is most important in altering the phenotypic characteristics under specific circumstances, it should be possible to identify who might and might not benefit from exaggerated intakes of this trace element and during what periods of life that efficacy might be greatest. The characterization of the specific molecular target(s) for this and other nutrients represents a major hurdle for the science of nutrition. Nevertheless, it is fundamental to truly understanding the involvement of nutrients in cancer prevention as well as in other health anomalies.

Another nutrient with apparent significance in the cancer process is folate (folic acid). Its essential role in the de novo biosynthesis of purines and pyrimidines, and thus in DNA replication and cell division, and for the synthesis of $S$-adenosylmethionine, a methyl donor for more than 100 biochemical reactions including methylation of DNA, places it in a unique position relative to DNA stability (Kim et al. 1997; Molloy \& Scott, 2001). These biosynthetic pathways, each of which is important to DNA metabolism, appear to compete when the dietary methyl supply is inadequate, as in folate deficiency, possibly resulting in altered DNA methylation (an epigenetic event), disruption of DNA integrity, disruption of DNA repair and, consequently, increased risk for several disorders (Molloy \& Scott, 2001). Hypomethylation and DNA strand breaks arising from folate inadequacy may actually promote the incorporation of viruses such as human papilloma virus into human DNA (Choi \& Mason, 2000). Additional research is need to determine if the response to folate inadequacy is also observed when methyl donor supply is suppressed by depression in choline, vitamin $\mathrm{B}_{12}$, pyridoxine and a variety of other nutrients.

A number of non-essential phytonutrients have also been found to impact health (Clydesdale, 1998; Milner, 2000). Recent studies have revealed that the use of transgenic and knockout animals offers exciting opportunities to define the mechanisms by which nutrients including phytochemicals function. Diethiolethione represents one class of nutrients that has been reported to influence a variety of molecular targets associated with cancer (Kensler et al. 2000). One of the major mechanisms of protection against carcinogenesis, mutagenesis and other forms of toxicity mediated by carcinogens is the induction of enzymes involved in their metabolism, particularly phase 2 enzymes such as glutathione S-transferases, UDP-glucuronosyl transferases and quinone reductases. The use of a knockout animal model has revealed that 1,2-dithiole-3-thione triggers nuclear accumulation of the transcription factor $\mathrm{Nrf} 2$ and its enhanced binding to the antioxidant response element, leading to transcriptional activation of a score of genes involved in carcinogen detoxification and attenuation of oxidative stress (Ramos-Gomez et al. 2001). More recently, Gupta et al. (2001) found that polyphenols in green tea were effective in reducing the incidence and metastasis in the transgenic adenocarcinoma mouse prostate model and increased the overall longevity of these animals. Additional studies are needed that employ a range of gene expressions to determine what impact genetic backgrounds have on the response to individual nutrients.

Foods and food components may also influence the microenvironment within the gastrointestinal tract. Inulin and oligofructose are intriguing dietary fermentable fibres that may have an impact on a number of processes directly and indirectly, and thereby influence health (Roberfroid, 1993). Inulin and oligofructose are fructans with a degree of polymerization of 2 to 60 and 2 to 20, respectively. Owing to their structural conformation, they are resistant to hydrolysis by human alimentary enzymes and therefore are fermented almost exclusively by colonic bifidobacteria and bacteroides. This fermentation increases faecal bacterial biomass, decreases caeco-colonic $\mathrm{pH}$ and produces a large amount of fermentation products, among which are short-chain fatty acids. While the long-term implications of changing the intestinal microflora remain unknown, the changes are consistent with an induction of genes in these micro-organisms and with a reduction in gastrointestinal distress (Roberfroid, 1993; Kleessen et al. 2001).

The ability of several nutrients to influence the same biological processes, as mentioned above, raises issues about possible synergy, as well as antagonistic interactions, among dietary components. Future studies must characterize nutrients in terms of their relative effectiveness, dose dependency, temporality, consistency and specificity. The defining of diet-specific molecular targets in terms of genetic and epigenetic events that lead to phenotypic changes should assist in the development of new and creative dietary intervention strategies for not only reducing diseases but also improving the overall quality of life.

\section{Dynamics between biomarkers and long-term intervention}

Unquestionably, scientifically sound and probing intervention studies must be viewed as the cornerstone for developing nutrition guidance for individuals. Regrettably, the sheer number of long-term intervention studies that will be needed to define nutrient interactions will surely be impractical in terms of speed of discovery and overall cost to society. Alternative procedures will thus be required to predict benefit and risk of selected interventions. These approaches will necessitate the use of sensitive and reliable biomarkers. Factors similar to those evaluated by environmental toxicologists (Suk \& Collman, 1988; Sakai, 2000) will be needed to evaluate the benefits/risks of functional foods and/or their components. Fundamental to this process will be biomarkers that evaluate: (1) the bioactive food component capable of modifying a molecular target (intake/exposure biomarker), (2) biological responses that evaluate directly or indirectly disease risk or health maintenance (effect biomarker), and (3) factors modifying the response such as genetic and the environment (susceptibility biomarker; Milner, 1999). To assess adequately whether a food or its component has a physiological 
effect it is imperative that stringent experimental design characteristics are followed. Several factors, including appropriateness of controls, randomization of subjects, blinding, statistical power of study, presence of bias, attrition rates, recognition and control of confounding factors (e.g. weight change or nutrition status) and appropriateness of statistical tests and comparisons, are addressed in a guidance document published in the Federal Register (1999). Each of these factors must become the mainstay for all investigations. Pre-clinical investigations will require that many if not most of these same factors be considered in their experimental design. Whatever biomarkers are established, it is clear that they must be readily accessible, easily and reliably assayed, differentially expressed in normal and diseased conditions, directly associated with disease progression, modifiable and, most important, 'predictive' (Fig. 1). Similar to the US Department of Agriculture's pyramid that is used for dietary guidance, it is likely that the early predicative biomarkers will not be at the top because of the lateness of the observation but be focused at the base where they will be more specific and timelier. Thus, the future of nutritional biomarkers is likely to reside in the enhanced use of molecular technologies to help decode who will and will not benefit from intervention strategies and, just as important, who might be placed at harm.

\section{Genetic polymorphisms and dietary variability}

Increasing genetic polymorphisms are thought to have a role in the ability of individuals to withstand exposure to exogenous carcinogens or to inhibit initiation, promotion or proliferation in carcinogenesis. It is certainly plausible that polymorphic differences have been a contributing

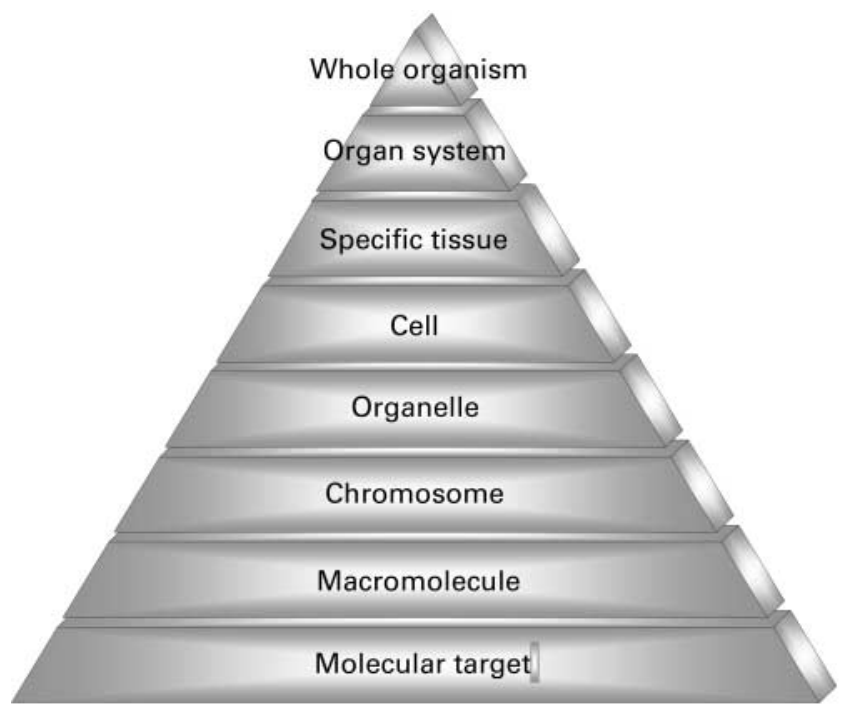

Fig. 1. Priority of potential nutritional biomarkers for evaluating the health benefits associated with bioactive food components. The top of the pyramid should be used sparingly while the molecular target base should be used extensively. Regardless of the biomarker used, it should ideally be readily accessible, easily and reliably assayed, differentially expressed in normal and abnormal conditions, directly associated with progression of the abnormal condition, modifiable and, most importantly, predictive. factor in the inconsistencies surrounding dietary components and health (Cotton et al. 2000). For example, in the Alpha-Tocopherol, Beta-Carotene Cancer Prevention Study (ATBC Study), there was a low prevalence of polymorphisms in genes coding for activation (phase I) enzymes CYP1A1 (0.07) and CYP2E1 (0.02) and a high prevalence in genes coding for detoxification (phase II) enzymes GSTM1 (0.40) and NQO1 (0.20) (Woodson et al. 1999). Seven of ten members of this sample carried the VDR-Taq1 polymorphism (t) associated with lower risk for prostate cancer, which may account in part for lower cancer rates in Finland compared with the United States (Woodson et al. 1999). Further, in a nested casecontrol study within the ATBC Study, glutathione peroxidase 1 (hGPX1), an Se-dependent enzyme involved in detoxification of hydrogen peroxide, was found to have a polymorphism exhibiting a proline to leucine replacement at codon 198. This polymorphism conferred a relative risk for lung cancer risk of 1.8 for heterozygotes and 2.3 for homozygous variants, compared with homozygote wild types (Ratnasinghe et al. 2000).

\section{The future}

Research in nutrition and health in this new millennium must give top priority to studies that will aid in understanding the basic molecular events by which nutrients influence biological process. A well-coordinated, multidisciplinary effort among scientists - including nutritional scientists, molecular biologists, geneticists, statisticians and clinical cancer researchers - may be needed to advance this molecular approach to nutrition-related cancer research. Many research questions and issues will need to be addressed for this approach to become a reality, including the minimum intake of bioactive components to bring about phenotypic change and how genetically controlled processes, including acclimatization, may change the overall response. While the challenges to researchers will be enormous, the potential rewards in terms of morbidity and mortality could also be enormous.

\section{References}

Abdulla M \& Gruber P (2000) Role of diet modification in cancer prevention. Biofactors 12, 45-51.

Aldana SG (2001) Financial impact of health promotion programs: a comprehensive review of the literature. American Journal for Health Promotion 15, 296-320.

Awad AB \& Fink CS (2000) Phytosterols as anticancer dietary components: evidence and mechanism of action. Journal of Nutrition 130, 2127-2130.

Blanchard RK, Moore JB, Green CL \& Cousins RJ (2001) Modulation of intestinal gene expression by dietary zinc status: effectiveness of cDNA arrays for expression profiling of a single nutrient deficiency. Proceedings of the National Academy of Sciences USA 98, 13507-13513.

Bradlow HL, Telang NT, Sepkovic DW \& Osborne MP (1999) Phytochemicals as modulators of cancer risk. Advances in Experimental Medicine and Biology 472, 207-221.

Chapkin RS, Fan Y \& Lupton JR (2000) Effect of diet on colonicprogrammed cell death: molecular mechanism of action. Toxicology Letters 112-113, 411-414. 
Choi SW \& Mason JB (2000) Folate and carcinogenesis: an integrated scheme. Journal of Nutrition 130, 129-132.

Clark LC, Combs GF Jr, Turnbull BW, Slate EH, Chalker DK, Chow J, Davis LS, Glover RA, Graham GF, Gross EG, Krongrad A, Lesher JL Jr, Park HK, Sanders BB Jr, Smith CL \& Taylor JR (1996) Effects of selenium supplementation for cancer prevention in patients with carcinoma of the skin. A randomized controlled trial. Nutritional Prevention of Cancer Study Group. Journal of the American Medical Association 276, 1957-1963.

Clarkson PM \& Thompson HS (2000) Antioxidants: what role do they play in physical activity and health? American Journal of Clinical Nutrition 72, Suppl. 2, 637S-646S.

Clydesdale FM (1998) Science, education, and technology: new frontiers for health. Critical Reviews in Food Science and Nutrition 38, 397-419.

Cotton SC, Sharp L, Little J \& Brockton N (2000) Glutathione S-transferase polymorphisms and colorectal cancer: a HuGE review. American Journal of Epidemiology 151, 7-32.

DellaPenna D (1999) Nutritional genomics: manipulating plant micronutrients to improve human health. Science 285, 375-379.

Diplock AT, Charleux JL, Crozier-Willi G, Kok FJ, Rice-Evans C, Roberfroid M, Stahl W \& Vina-Ribes J (1998) Functional food science and defence against reactive oxidative species. British Journal of Nutrition 80, Suppl. 1, S77-S112.

Eisenberg DM, Davis RB, Ettner SL, Appel S, Wilkey S, Van Rompay M \& Kessler RC (1998) Trends in alternative medicine use in the United States, 1990-1997: results of a follow-up national survey. Journal of the American Medical Association 80, 1569-1575.

Fafournoux P, Bruhat A \& Jousse C (2000) Amino acid regulation of gene expression. Biochemistry Journal 351, 1-12.

Federal Register (1999) Guidance for industry: significant scientific agreement in the review of health claims for conventional foods and dietary supplements. Federal Register 64 71794.

Gilbert L (1999) 1999 Health Focus Trend Report. DesMoines, IA: HealthFocus.

Gill HS \& Cross ML (2000) Anticancer properties of bovine milk. British Journal of Nutrition 84, Suppl. 1, S161-S166.

Go VL, Wong DA \& Butrum R (2001) Diet, nutrition and cancer prevention: where are we going from here? Journal of Nutrition 131, Suppl. 11, 3121S-3126S.

Gray T, Koo J \& Nettesheim P (2001) Regulation of mucous differentiation and mucin gene expression in the tracheobronchial epithelium. Toxicology 160, 35-46.

Greenwald P \& Milner JA (2001) Nutrition: a major player in chronic diseases. The Chronicle of Higher Education, B15

Guarente L \& Kenyon C (2000) Genetic pathways that regulate ageing in model organisms. Nature 408, 255-262.

Gupta S, Hastak K, Ahmad N, Lewin JS \& Mukhtar H (2001) Inhibition of prostate carcinogenesis in TRAMP mice by oral infusion of green tea polyphenols. Proceedings of the National Academy of Sciences USA 98, 10350-10355.

Hasler CM, Kundrat S \& Wool D (2000) Functional foods and cardiovascular disease. Current Atherosclerosis Reports 2, 467-475.

Hegele RA (1998) A review of intestinal fatty acid binding protein gene variation and the plasma lipoprotein response to dietary components. Clinical Biochemistry 31, 609-612.

Hegelea RA, Jugenberg M, Connelly PW \& Jenkins DJA (1997) Evidence for gene-diet interaction in the response of blood pressure to dietary fibre. Nutrition Research 17, $1229-1238$.

Hilakivi-Clarke L, Cho E, Onojafe I, Raygada M \& Clarke R (1999) Maternal exposure to genistein during pregnancy increases carcinogen-induced mammary tumorigenesis in female rat offspring. Oncology Report 6, 1089-1095.

Issa JP (2000) CpG-island methylation in aging and cancer. Current Topics in Microbiology and Immunology 249, $101-118$.

Jackson AA (2000) Nutrients, growth, and the development of programmed metabolic function. Advances in Experimental Medicine and Biology 478, 41-55.

Kensler TW, Curphey TJ, Maxiutenko Y \& Roebuck BD (2000) Chemoprotection by organosulfur inducers of phase 2 enzymes: dithiolethiones and dithiins. Drug Metabolism and Drug Interactions 17, 3-22.

Kim YI, Pogribny IP, Basnakian AG, Miller JW, Selhub J, James SJ \& Mason JB (1997) Folate deficiency in rats induces DNA strand breaks and hypomethylation within the p53 tumor suppressor gene. American Journal of Clinical Nutrition 65, $46-52$.

Kleessen B, Hartmann L \& Blaut M (2001) Oligofructose and long-chain inulin: influence on the gut microbial ecology of rats associated with a human faecal flora. British Journal of Nutrition 86, 291-300.

Knowles LM \& Milner JA (2000) Allyl sulfides modify cell growth. Drug Metabolism and Drug Interactions 17, $81-107$.

Leclercq C, Valsta LM \& Turrini A (2001) Food composition issues - implications for the development of food-based dietary guidelines. Public Health Nutrition 4, 677-682.

Lee TH \& Pelletier J (2001) Functional characterization of WT1 binding sites within the human vitamin $\mathrm{D}$ receptor gene promoter. Physiological Genomics 7, 187-200.

Merrill AH Jr, Sullards MC, Wang E, Voss KA \& Riley RT (2001) Sphingolipid metabolism: roles in signal transduction and disruption by fumonisins. Environmental Health Perspectives 109, Suppl. 2, 283-289.

Milner JA (1999) Functional foods and health promotion. Journal of Nutrition 129, Suppl. 7, 1395S-1397S.

Milner JA (2000) Functional foods: the US perspective. American Journal of Clinical Nutrition 71, Suppl. 6, 1654S-1659S.

Molloy AM \& Scott JM (2001) Folates and prevention of disease. Public Health Nutrition 4, 601-609.

National Center for Health Statistics (1997) Report of final mortality statistics, 1995. Monthly Vital Statistics Report 45, Suppl. 2(11), 12 June.

Paabo S (2001) Genomics and society. The human genome and our view of ourselves. Science 291, 1219-1220.

Pan DA, Mater MK, Thelen AP, Peters JM, Gonzalez FJ \& Jump DB (2000) Evidence against the peroxisome proliferatoractivated receptor alpha (PPARalpha) as the mediator for polyunsaturated fatty acid suppression of hepatic L-pyruvate kinase gene transcription. Journal of Lipid Research 41, $742-751$.

Potter JD (1997) Cancer prevention: epidemiology and experiment. Cancer Letters 114, 7-9.

Prosky L (2000) When is dietary fiber considered a functional food? Biofactors 12, 289-297.

Ramos-Gomez M, Kwak MK, Dolan PM, Itoh K, Yamamoto M, Talalay P \& Kensler TW (2001) From the cover: sensitivity to carcinogenesis is increased and chemoprotective efficacy of enzyme inducers is lost in nrf2 transcription factor-deficient mice. Proceedings of the National Academy of Sciences USA 98, 3410-3415.

Rapuri PB, Gallagher JC, Kinyamu HK \& Ryschon KL (2001) Caffeine intake increases the rate of bone loss in elderly women and interacts with vitamin D receptor genotypes. American Journal of Clinical Nutrition 74, 569-570.

Ratnasinghe D, Tangrea JA, Andersen MR, Barrett MJ, Virtamo J, Taylor PR \& Albanes D (2000) Glutathione peroxidase 
codon 198 polymorphism variant increases lung cancer risk. Cancer Research 60, 6381-6383.

Roberfroid M (1993) Dietary fiber, inulin, and oligofructose: a review comparing their physiological effects. Critical Reviews in Food Science and Nutrition 33, 103.

Roberfroid MB (2000) Concepts and strategy of functional food science: the European perspective. American Journal of Clinical Nutrition 71, Suppl. 6, 1660S-1664S.

Ross JA (2000) Dietary flavonoids and the MLL gene: a pathway to infant leukemia? Proceedings of the National Academy of Sciences USA 97, 4411-4413.

Sakai T (2000) Biomarkers of lead exposure. Industrial Health 38, $127-142$.

Schrauzer GN (2000) Anticarcinogenic effects of selenium. Cellular and Molecular Life Sciences 57, 1864-1867.
Suk WA \& Collman GW (1988) Genes and the environment: their impact on children's health. Environmental Health Perspectives 106, 817-820.

Turturro A, Blank K, Murasko D \& Hart R (1994) Mechanisms of caloric restriction affecting aging and disease. Annals of the New York Academy of Science 719, 159-170.

Ward WE, Jiang FO \& Thompson LU (2000) Exposure to flaxseed or purified lignan during lactation influences rat mammary gland structures. Nutrition and Cancer 37, $187-192$.

Woodson K, Ratnasinghe D, Bhat NK, Stewart C, Tangrea JA, Hartman TJ, Stolzenberg-Solomon R, Virtamo J, Taylor PR \& Albanes D (1999) Prevalence of disease-related DNA polymorphisms among participants in a large cancer prevention trial. European Journal of Cancer Prevention 8, 441-447. 